\title{
Visible reconstruction by a circular holographic display from digital holograms recorded under infrared illumination
}

\author{
E. Stoykova, ${ }^{1,3,5, *}$ F. Yaraş, ${ }^{1,4}$ H. Kang, ${ }^{1,3}$ L. Onural, ${ }^{1}$ A. Geltrude, ${ }^{2}$ M. Locatelli, ${ }^{2}$ \\ M. Paturzo, ${ }^{2}$ A. Pelagotti, ${ }^{2}$ R. Meucci, ${ }^{2}$ and P. Ferraro ${ }^{2}$ \\ ${ }^{1}$ Department of Electrical and Electronics Engineering, Bilkent University TR-06800 Ankara, Turkey \\ ${ }^{2}$ Consiglio Nazionale delle Richerche-Instituto Nazionale di Ottica Via Campi Flegrei 34, 80078 Napoli and Largo Fermi 6, 50125 Firenze, Italy \\ ${ }^{3}$ Currently at Korea Electronics Technology Institute (KETI), 8 Floor, \#1599 Sangam-dong, Mapo-gu, Seoul 121-835, South Korea \\ ${ }^{4}$ Currently at Qualcomm Inc., 100 Burtt Road, Suite 123, Andover, Massachusetts 01810, USA \\ ${ }^{5}$ Bulgarian Academy of Sciences, 109 Acad. Georgi Bonchev, 1113 Sofia, Bulgaria \\ ${ }^{*}$ Corresponding author: estoykova@iomt.bas.bg
}

Received April 5, 2012; revised June 5, 2012; accepted June 5, 2012;

posted June 7, 2012 (Doc. ID 166025); published July 20, 2012

\begin{abstract}
A circular holographic display that consists of phase-only spatial light modulators is used to reconstruct images in visible light from digital holograms recorded under infrared $(10.6 \mu \mathrm{m})$ illumination. The reconstruction yields a holographic digital video display of a three-dimensional ghostlike image of an object floating in space where observers can move and rotate around it. (c) 2012 Optical Society of America

OCIS codes: $\quad 090.1995,090.2870,090.4220,110.3080$.
\end{abstract}

Multiview capture of holograms together with a holographic display built from many spatial light modulators (SLMs) widens the range of applications of digital holography [는 $]$. A circular holographic display [ㄴ,5] puts less severe requirements to the space-bandwidth product of the system and supports full parallax binocular vision at an increased viewing angle. With such a display system, observers can see three-dimensional (3D) ghostlike images floating in space and can move and rotate around them. Successful dynamic wide-angle optical reconstruction from computer-generated holograms using a circular holographic display built from nine phase-only SLMs has been recently reported [5]. However, if the data fed to the SLMs are retrieved from digitally recorded holograms, the reconstruction is no longer a trivial task. The main challenge is the difference in coding parameters of the recording and display systems. Here, we demonstrate wide-angle optical reconstruction using the circular holographic display in [5] under illumination at $0.532 \mu \mathrm{m}$ from a set of holograms recorded at $10.6 \mu \mathrm{m}$. For such a case, the differences in the pixel geometries of the light-sensitive areas of the capture and display systems and between the wavelengths of holographic recording and optoelectronic reconstruction strongly alter the reconstruction distance and the lateral and longitudinal dimensions of the reconstruction volume.

The interest in capturing holograms in the longwavelength infrared (IR) range arises from such valuable features of IR digital holography as shorter recording distances, larger viewing angles, high output powers of IR laser sources, and less stringent requirements on the stability of the interferometric system. Furthermore, transparency of many materials in the IR range makes inspection of such components using long-wavelength IR holograms feasible. The recent advances in digital image sensors, such as pyrocameras and focal plane array microbolometers with a pixel size of $25 \mu \mathrm{m}$ and no need of cryogenic cooling, have further improved IR digital holography [6-8]. Digital holography at $10.6 \mu \mathrm{m}$, both in transmission and reflection configurations, for optical reconstruction of objects with sizes of less than $1 \mathrm{~mm}$ to $40 \mathrm{~cm}$ was recently investigated [9]. A nondistorted single SLM visualization, at $0.532 \mu \mathrm{m}$, from a hologram of a bronze reproduction of the Benvenuto Cellini Perseus sculpture with a height of $33 \mathrm{~cm}$ was presented in [10]. The hologram was recorded at $10.6 \mu \mathrm{m}$ in a Fourier configuration. The statuette was a large object with regard to the common digital holography restrictions. For comparison, in this work, we used the same test object, but with a modified setup for off-axis recording, as shown in Fig. 1. We used a $110 \mathrm{~W} \mathrm{CW} \mathrm{CO}_{2}$ laser, emitting at $10.6 \mu \mathrm{m}$ and operating at $\mathrm{TEM}_{00}$ fundamental mode. We used only a fraction $(30 \mathrm{~W})$ from the full power of the laser. The laser beam had a waist of $10 \mathrm{~mm}$ and a divergence of $2 \mathrm{mrad}$. For capture from different perspectives, the object was rotated with an angular step of $3^{\circ}$. The holograms were acquired by means of an ASi (amorphous silicon) thermal camera (Thermoteknix MIRICLE 307K) with $n_{x} \times n_{y}=$ $640 \times 480$ pixels with $\Delta_{1}=25 \mu \mathrm{m}$ pixel period. The maximum angle, $\theta_{\max }$, between the reference and the object beams to satisfy the Wittaker-Shannon sampling requirement for a given $\Delta_{1}$ is found from $\sin \left(\frac{\theta_{\max }}{2}\right)=\frac{\lambda_{1}}{4 \Delta_{1}}$, where

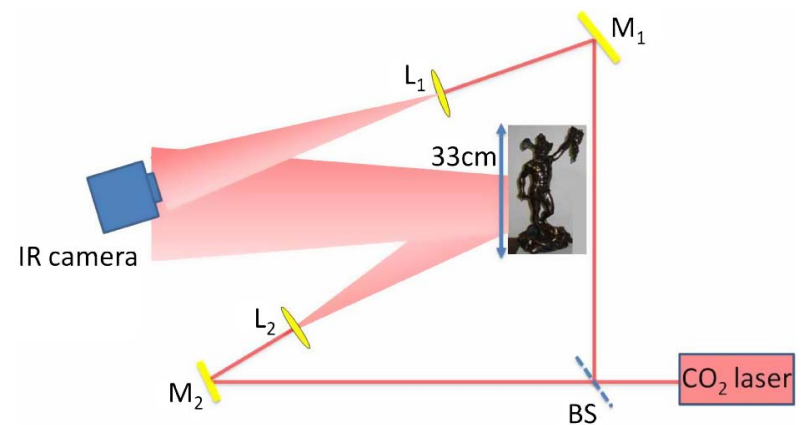

Fig. 1. (Color online) Experimental setup for the recording of IR holograms [10]. 
$\lambda_{1}$ is the wavelength of recording. The minimum distance between the object and the sensor, which is proportional to $\Delta_{1} D / \lambda_{1}$ if the object lateral size $D$ is much greater than the sensor size, decreases substantially in the longwavelength IR range. For the scheme in Fig. 1, the distance between the object and the camera was $z_{0}=$ $880 \mathrm{~mm}$. The expanding reference beam had a spherical wavefront $R_{1}(x, y)=\exp \left[-i \frac{\pi}{\lambda_{1}} \frac{\left(x^{2}+y^{2}\right)}{r_{1}}\right]$ with a radius of curvature $r_{1}=z_{0} / 2$. Here, $\left(x=p \Delta_{1}, y=q \Delta_{1}, p=\right.$ $\left.1 \ldots n_{x}, q=1 \ldots n_{y}\right)$ are the coordinates in the plane of the sensor aperture. All spherical waves are given in paraxial approximation.

In the case of reconstruction with a SLM with a pixel period $\Delta_{2}$, the recorded hologram undergoes a linear stretching with a coefficient $m=\Delta_{2} / \Delta_{1}$. At illumination with a wavelength $\lambda_{2}$, the value of the angle $\theta_{\max }$ remains unchanged, if $\Delta_{1} / \Delta_{2}=\lambda_{1} / \lambda_{2}$ is fulfilled. This would require $\Delta_{2}=1.25 \mu \mathrm{m}$ for a reconstruction at $0.532 \mu \mathrm{m}$. For illumination at $\lambda_{2}$ with a spherical wavefront with a radius of curvature $r_{2}$, the distance $z_{i}$ at which the reconstructed image is in focus is given by $\frac{1}{z_{i}}=\frac{1}{r_{2}} \pm \frac{\mu}{m^{2}}\left(\frac{1}{z_{0}}-\frac{1}{r_{1}}\right)$, where $\mu=\lambda_{1} / \lambda_{2}[10,11]$. For the scheme in Fig. 1, the formula gives $z_{i}=1.78 \mathrm{~m}$ for a reconstruction with $\Delta_{2}=8 \mu \mathrm{m}$ at illumination with a plane wave $\left(r_{2} \rightarrow \infty\right)$ with longitudinal magnification $M_{\text {long }}=\frac{d z_{i}}{d z_{o}}=\left(\frac{z_{i}}{z_{o}}\right)^{2} \frac{\mu}{m^{2}}=$ $m^{2} \frac{\lambda_{1}}{\lambda_{2}}=2.04$, where we substitute $z_{i}=z_{o} \frac{m^{2}}{\mu}$. The lateral magnification, given by $M_{\text {lat }}=\frac{\mu}{m} \frac{z_{i}}{z_{0}}$, is equal to $M_{\text {lat }}=$ $m=0.32$ for plane wave illumination. Figure 2 presents the optical reconstruction under plane wave illumination for one of the recorded holograms. We applied spatial filtering to suppress the zero-order and twin-image terms [12] and retrieved the object wave by a multiplication of the filter output in the spatial domain with a numerical reference wave $R_{2}^{*}(\xi, \eta)=\exp \left[-i \frac{\pi}{\lambda_{2}} \frac{\left(\xi^{2}+\eta^{2}\right)}{r_{2}^{\prime}}\right]$. Here, $(\xi=$ $\left.p \Delta_{2}, \eta=q \Delta_{2}, p=1 \ldots N_{x}, q=1 \ldots N_{y}\right)$ are the coordinates in the plane of the SLM, and $r_{2}^{\prime}=\frac{z_{i}}{2}=\frac{z_{o} m^{2}}{2 \mu}$. The asterisk denotes the complex conjugate. The phase of the retrieved object wave was fed into a Holoeye HEO1080P phase-only liquid-crystal-on-silicon (LCoS) SLM with $N_{x} \times N_{y}=1920 \times 1080$ pixels and $\Delta_{2}=8 \mu \mathrm{m}$. The retrieved phase distribution with $n_{x} \times n_{y}=640 \times 480$
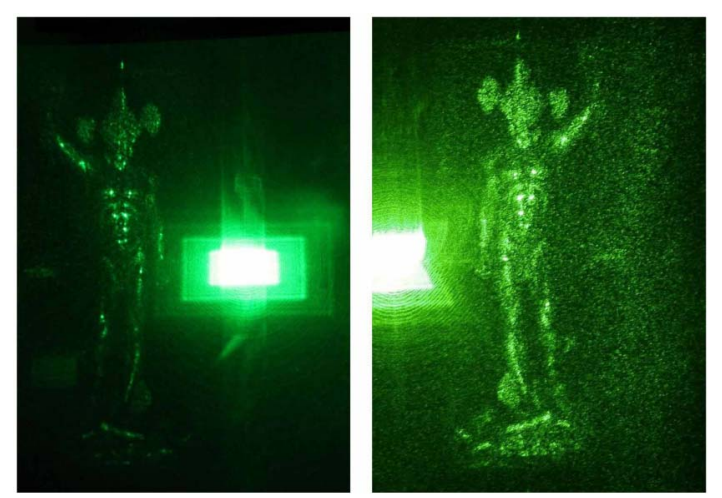

Fig. 2. (Color online) Left, SLM optical reconstruction at $0.532 \mu \mathrm{m}$ of the hologram captured at $10.6 \mu \mathrm{m}$ projected on a diffuse screen. Right, ghostlike SLM optical reconstruction of the same hologram.

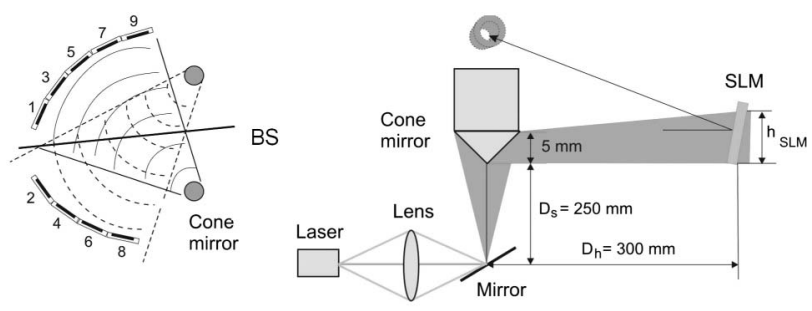

Fig. 3. Circular holographic display. Left, arrangement of the nine phase-only SLMs, denoted as 1...9. Right, illumination of a single SLM [5].

pixels was placed at the center of the SLM. The phase of $R_{2}^{*}(\xi, \eta)$ was added to the SLM's pixels around the hologram to focus the rays reflected by them outside the viewing zone. Figure $\underline{2}$ gives the photographs of the reconstruction observed on a diffuse screen and of the 3D image floating in space.

The multiview optoelectronic reconstruction of the recorded nine holograms was made with a holographic video display system built from nine phase-only Holoeye HEO-1080P SLMs that formed a circular configuration [5]. Elimination of the gaps between the SLMs was provided by a beam splitter to tile them side by side (Fig. 3) [5] and to achieve a virtual alignment with a continuous increased field of view. To position the reconstructed 3D image slightly above the display setup and to avoid blocking of the observer's vision by the display's components, the SLMs were also tilted up at a small angle. Negligible reduction in the quality of the reconstructions for a tilted illumination of up to $20^{\circ}$ has been shown by experiments and subjective test results [13]. All SLMs were illuminated with a single astigmatic expanding wave by means of a cone mirror [5]:

$$
W(\xi, \eta)=\exp \left(-i k_{2} \frac{\xi^{2}}{D_{h}}\right) \exp \left[-i \frac{k_{2}}{2} \frac{\left(\eta+\frac{h_{\mathrm{SLM}}}{2}\right)^{2}}{D_{h}+D_{s}}\right],
$$

where $k_{2}=2 \pi / \lambda_{2}, D_{h}$ is the distance between the axis of the cone mirror and the SLM, $h_{\mathrm{SLM}}$ is the height of the SLM, and $D_{s}$ is the distance between the apex of the cone mirror and the point source of the wave positioned on the line of the cone mirror axis.

The hologram computation for each of the SLMs consisted of two steps: (i) retrieval of the phase distribution $\Psi(\xi, \eta)$ in the complex amplitude $a(\xi, \eta) \exp \left[i k_{2} \Psi(\xi, \eta)\right]$ of the object field from the recorded off-axis 8 bit encoded digital hologram; (ii) compensation for the nonsymmetrical illumination with the cone mirror and adjustment of the reconstruction volume position. The first step implied spatial filtering, and then we discarded the amplitude $a(\xi, \eta)$ as we used phase-only SLMs. Improvement of image reconstruction was observed if the spectrum of the phase-only term $\exp \left[i k_{2} \Psi(\xi, \eta)\right] R_{2}(\xi, \eta)$ was filtered again with the same filter, as presented in Fig. 2. Finally, we multiplied the second filter output by $R_{2}^{*}(\xi, \eta)$ in the spatial domain to obtain the object field as $H(\xi, \eta)=$ $\exp \left[i k_{2} \Psi(\xi, \eta)\right]$. The distance between the reconstruction volume and each SLM was $35 \mathrm{~cm}$. To compensate for the nonsymmetrical illumination, we computed the phase of $H(\xi, \eta) W^{*}(\xi, \eta)$. Under plane wave illumination, $z_{i}$ ex- 

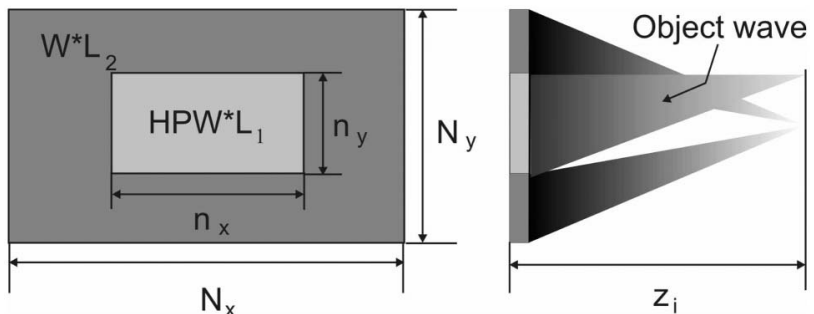

Fig. 4. Front and side views of each SLM with the hologram and the noninformative zone; the object wave focuses above the focus of rays from the noninformative pixels under illumination with the astigmatic wave $\mathrm{W}$.
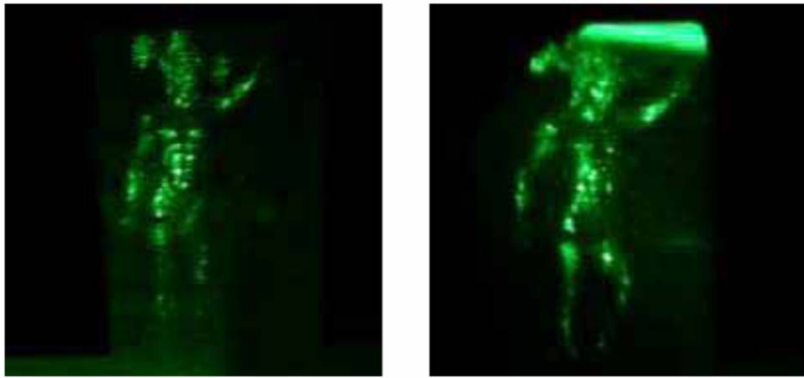

Fig. 5. (Color online) Ghostlike SLM multiview optical reconstruction at $0.532 \mu \mathrm{m}$ from holograms captured at $10.6 \mu \mathrm{m}$. Left, view at $12^{\circ}$. Right, image from a video taken with a camera that rotates around the reconstructed image (Media 1) .

ceeded $35 \mathrm{~cm}$, and we introduced a digital converging lens term $L_{1}(\xi, \eta)=\exp \left[i k_{2}\left(\xi^{2}+\eta^{2}\right) / \rho_{1}\right]$ with a focal distance $\rho_{1}=43.5 \mathrm{~cm}$. To separate the image from the strong nondiffracted beam due to the pixelated nature of SLMs (Fig. 2) [5], we multiplied the hologram area with $P(\eta)=\exp \left(i \bar{k}_{2} \eta \sin \theta_{t}\right)$, where $\theta_{t}=2^{\circ}$. The holograms were placed at the centers of the SLMs (Fig. 4). A converging lens term $L_{2}(\xi, \eta)=\exp \left[i k_{2}\left(\xi^{2}+\eta^{2}\right) / \rho_{2}\right]$, with $\rho_{2}=35 \mathrm{~cm}$, was introduced in addition to $W^{*}(\xi, \eta)$ for the pixels outside the hologram to gather the light reflected from them below the reconstructed image. The magnification of the reconstruction volume in the longitudinal and lateral directions was more or less the same: $M_{\text {long }}=0.078$ and $M_{\text {lat }}=0.062$. Figure $\underline{5}$ presents the reconstruction at $12^{\circ}$ and the video showing the ghostlike image captured with a camera that rotates around it (Media 1). An optical lens was used to blend reconstructions more smoothly. The quality of the image is good, especially in view of the small number of pixels in the recorded holograms. Rather small details are easily recognizable with a smooth parallax within a viewing angle of $24^{\circ}$.

In conclusion, we obtained an optical reconstruction with a circular display consisting of nine Holoeye LCoS spatial light modulators (pixel period $8 \mu \mathrm{m}$ ) under illumination with a $0.532 \mu \mathrm{m}$ wavelength. The reconstruction is obtained from a set of nine holograms, which were captured at a 20 times larger wavelength. The results show good quality reconstructed ghostlike images for a continuously varying parallax within a $24^{\circ}$ viewing angle, paving the way for a reliable multiview IR-recording/ visible-light reconstruction system. The presented holographic display can be used for virtual museum applications; it may also lead to holographic 3D displays for terahertz imaging.

This work is supported by the European Community (EC) within the Seventh Framework Programme (FP7) under Grant 216105 with the acronym Real 3D, and the Programma Operativo Nazionale (PON) project IT@CHA funded by the Italian Ministry of Education, University and Research (MIUR).

\section{References}

1. F. Yaraş, H. Kang, and L. Onural, Appl. Opt. 48, H48 (2009).

2. F. Yaraş, H. Kang, and L. Onural, J. Disp. Technol. 6, 443 (2010).

3. L. Onural, F. Yaraș, and H. Kang, Proc. IEEE 99, 576 (2011).

4. J. Hahn, H. Kim, Y. Lim, G. Park, and B. Lee, Opt. Express 16, 12372 (2008).

5. F. Yaras, H. Kang, and L. Onural, Opt. Express 19, 9147 (2011).

6. E. Allaria, S. Brugioni, S. De Nicola, P. Ferraro, S. Grilli, and R. Meucci, Opt. Commun. 215, 257 (2003).

7. S. De Nicola, P. Ferraro, S. Grilli, L. Miccio, R. Meucci, P. K. Buah-Bassuah, and F. T. Arecchi, Opt. Commun. 281, 1445 (2008).

8. A. Pelagotti, M. Locatelli, A. G. Geltrude, P. Poggi, R. Meucci, M. Paturzo, L. Miccio, and P. Ferraro, J. Disp. Technol. 6, 465 (2010).

9. A. Pelagotti, M. Paturzo, A. Geltrude, M. Locatelli, R. Meucci, P. Poggi, and P. Ferraro, 3D Research 1(4), 6 (2010).

10. M. Paturzo, A. Pelagotti, A. Finizio, L. Miccio, M. Locatelli, A. Gertrude, P. Poggi, R. Meucci, and P. Ferraro, Opt. Lett. 35, 2112 (2010).

11. R. Meier, J. Opt. Soc. Am. 55, 987 (1965).

12. E. Cuche, P. Marquet, and C. Depeursinge, Appl. Opt. 39, 4070 (2000).

13. F. Yaraş, "Three-dimensional holographic video display systems using multiple spatial light modulators," Ph.D. dissertation (Bilkent University, 2011). 